\title{
Resonant control of an atomic force microscope micro-cantilever for active $Q$ control
}

\author{
M. Fairbairna) and S. O. R. Moheimanib) \\ School of Electrical Engineering and Computer Science, The University of Newcastle, Callaghan, \\ New South Wales 2308, Australia
}

(Received 7 May 2012; accepted 29 July 2012; published online 22 August 2012)

\begin{abstract}
Active $Q$ control may be used to modify the effective quality $(Q)$ factor of an atomic force microscope (AFM) micro-cantilever when operating in tapping mode. The control system uses velocity feedback to obtain an effective cantilever $Q$ factor to achieve optimal scan speed and image resolution for the imaging environment and sample type. Time delay of the cantilever displacement signal is the most common method of cantilever velocity estimation. Spill-over effects from unmodeled dynamics may degrade the closed loop system performance, possibly resulting in system instability, when time delay velocity estimation is used. A resonant controller is proposed in this work as an alternate method of velocity estimation. This new controller has guaranteed closed loop stability, is easy to tune, and may be fitted into existing commercial AFMs with minimal modification. Images of a calibration grating are obtained using this controller to demonstrate its effectiveness. () 2012 American Institute of Physics. [http://dx.doi.org/10.1063/1.4746277]
\end{abstract}

\section{INTRODUCTION}

The atomic force microscope (AFM) (Ref. 1) can produce molecular and atomic resolution 3D images of sample surfaces by mapping the force interactions between a sharp probe tip located on the underside of a micro-cantilever and the sample surface. The ability of the AFM to image a wide variety of samples in air, ${ }^{2}$ vacuum, ${ }^{3}$ or liquid environments ${ }^{4}$ has made it a valuable tool in many fields of scientific research.

The sample surface is scanned in a raster pattern below the micro-cantilever with a piezoelectric scanner. When operating the AFM in contact mode the probe tip maintains constant contact with the sample at all times. Deflections of the cantilever are measured to obtain the sample height, which is plotted against the scan position to produce a 3D image of the sample surface.

Dragging the sample under the probe tip in constant contact may lead to damage of soft delicate samples due to lateral forces between the tip and the sample. To minimize lateral forces between the probe tip and the sample the AFM may be operated in amplitude modulation mode which is commonly referred to as tapping mode. ${ }^{5}$ When operating in tapping mode the cantilever is oscillated at a constant amplitude close to its first resonance frequency. The cantilever is oscillated by applying a sinusoidal voltage to a piezoelectric actuator positioned at the base of the cantilever. Cantilevers coated with a layer of piezoelectric material acting as an actuator are now becoming increasingly popular for tapping mode AFM, removing the need for the bulky actuator at the cantilever base. ${ }^{6}$

As the tip lightly taps the sample, once every oscillation cycle, the contact time between the tip and sample is

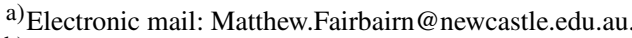

b) Electronic mail: Reza.Moheimani@newcastle.edu.au. decreased and consequently lateral forces are significantly reduced. ${ }^{7}$ For this reason tapping mode is the most popular imaging mode for biological samples. ${ }^{8,9}$

As the tip interacts with the sample surface the change in force between the tip and the sample, due to height changes in the sample topography, lead to a change in the effective stiffness of the cantilever ${ }^{5,10,11}$ resulting in a shift in the resonance frequency of the cantilever. This deviation in the resonance frequency causes a change in the oscillation amplitude $A(t)$, as shown in Fig. 1, which is proportional to the change in sample height. The change in $A(t)$ may therefore be measured to reflect changes in sample height.

Normal forces between the cantilever and sample may damage the sample or tip and may compress soft samples leading to inaccurate height measurements. ${ }^{7}$ For these reasons the tip-sample force is held constant, as the sample is scanned underneath the cantilever, by regulating the scanner in the vertical $(Z)$ direction using feedback control. A block diagram of the tapping mode $Z$ axis feedback control loop is shown in Fig. 2. The cantilever deflection is most commonly measured using the optical beam deflection technique ${ }^{12}$ which involves reflecting a laser beam off the surface of the cantilever onto a 4 quadrant photodiode sensor. Movement of the reflected beam on the photodiode sensor is representative of cantilever deflection. The oscillation amplitude is then demodulated from the displacement signal with an rms to dc converter or lock-in amplifier before being processed by the feedback controller. A feature in the sample topography is an input disturbance in the feedback loop. The output of the feedback controller is therefore proportional to this disturbance. This signal is used to measure the sample height as the sample is scanned underneath the controller. Sample topography is mapped by plotting this signal as a function of the horizontal position of the sample.

While tapping mode has an advantage over contact mode for imaging soft delicate samples it does have a drawback of 


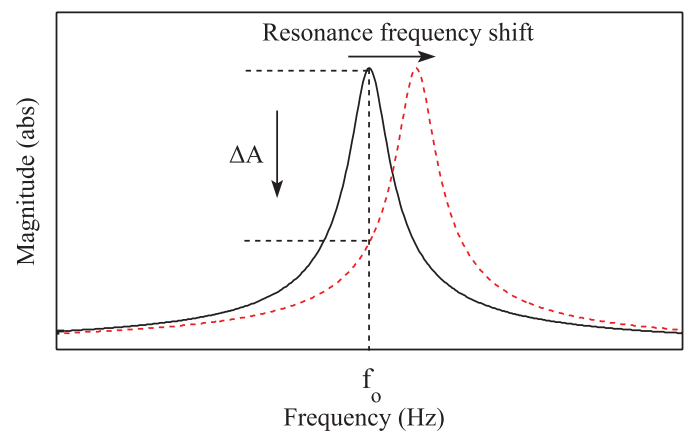

FIG. 1. Frequency response of the cantilever with no tip-sample interaction forces (-) and with tip-sample interaction forces (- -). The tip-sample interaction force causes a shift in resonance frequency leading to a change in oscillation amplitude of the cantilever oscillating at the frequency $f_{o}$.

a low imaging bandwidth. As the scan speed is increased the ability of the probe to track the sample topography is reduced. The bandwidth of the $Z$ axis feedback control loop determines the speed at which the probe can track the sample topography accurately.

The bandwidth of the $Z$ axis feedback loop is limited by the transient response of the cantilever. The transfer function of the cantilever in cascade with the demodulator is approximated by a low pass filter with a cutoff frequency of $\frac{\omega_{n}}{2 Q},{ }^{13}$ where $\omega_{n}$ is the natural frequency and $Q$ is the quality factor of the cantilever. $Q$ is inversely proportional to the cantilever damping coefficient $\zeta$, where $Q=\frac{1}{2 \zeta}$. The $Z$ axis controller is a PI controller in most commercial AFMs. The stability margins of the $Z$ axis feedback control loop are determined by the controller in cascade with the cantilever/demodulator. Increasing the bandwidth of the cantilever/demodulator transfer function will have the effect of widening the stability margins of the feedback loop allowing for an increase in the controller gain which leads to a broader bandwidth of the $Z$ axis feedback loop. The bandwidth of the cantilever/demodulator may be widened by increasing $\omega_{n}$ or reducing $Q$.

Increases in imaging bandwidth have been achieved through the use of smaller cantilevers ${ }^{14,15}$ which have higher resonance frequencies. The reduced length of the cantilever may limit the ability of the cantilever to track samples with large topographic features.

The effective $Q$ factor of the cantilever may be modified through the use of active $Q$ control. ${ }^{13,16}$ Sulchek et al. ${ }^{17,18}$ and
Fairbairn et al. ${ }^{19}$ have demonstrated that a reduction in the cantilever $Q$ factor using active $Q$ control improves the bandwidth of the $Z$ axis feedback loop allowing for faster scans of hard surfaced samples in air. Reducing the cantilever $Q$ factor may also be used to increase scan speeds when imaging in vacuum. ${ }^{20}$

Reducing the cantilever $Q$ factor results in a reduction in force sensitivity ${ }^{21}$ and an increase in tip-sample force ${ }^{22}$ which may not be desirable when imaging samples with fine features, soft fragile samples, and/or samples imaged in liquids.

The slope of the resonance curve for a cantilever with a high $Q$ factor will be steeper than that of a cantilever with a low $Q$ factor. Changes in oscillation amplitude due to changes in sample height will be larger for the high $Q$ cantilever. The high $Q$ cantilever will therefore have higher force sensitivity/resolution. ${ }^{23}$

Rodriguez and Garcia $^{24}$ determined that the average force between the cantilever tip and the sample is

$$
F_{T S} \propto \frac{k}{Q} \sqrt{\left(A_{0}^{2}-A_{s e t}^{2}\right)},
$$

where $k$ is the spring constant of the cantilever, $A_{0}$ is the free air oscillation amplitude of the cantilever, and $A_{\text {set }}$ is the setpoint oscillation amplitude of the cantilever. From (1) it can be easily seen that a cantilever with a high $Q$ factor will result in lower tip-sample forces. It is possible to decrease tapping forces by setting $A_{\text {set }}$ close to $A_{0}$. This will have no effect on force sensitivity and may introduce the problem of poor sample tracking in steep downward sloping regions of the sample..$^{25,26}$

The cantilever $Q$ factor may be increased with active $Q$ control. This is particularly useful when imaging a sample in liquid as the hydrodynamic forces acting on the cantilever may reduce its $Q$ factor by up to two orders of magnitude from that in air. ${ }^{27}$ Several researchers have demonstrated the benefits of increasing the cantilever $Q$ factor when imaging. Gao et al. $^{28}$ and Chen et al. ${ }^{29}$ obtained significant improvements in image quality when imaging DNA protein complexes in liquid.

\section{ACTIVE $Q$ CONTROL}

Active $Q$ control uses velocity feedback to modify the effective cantilever $Q$ factor. A reduction of the $Q$ factor

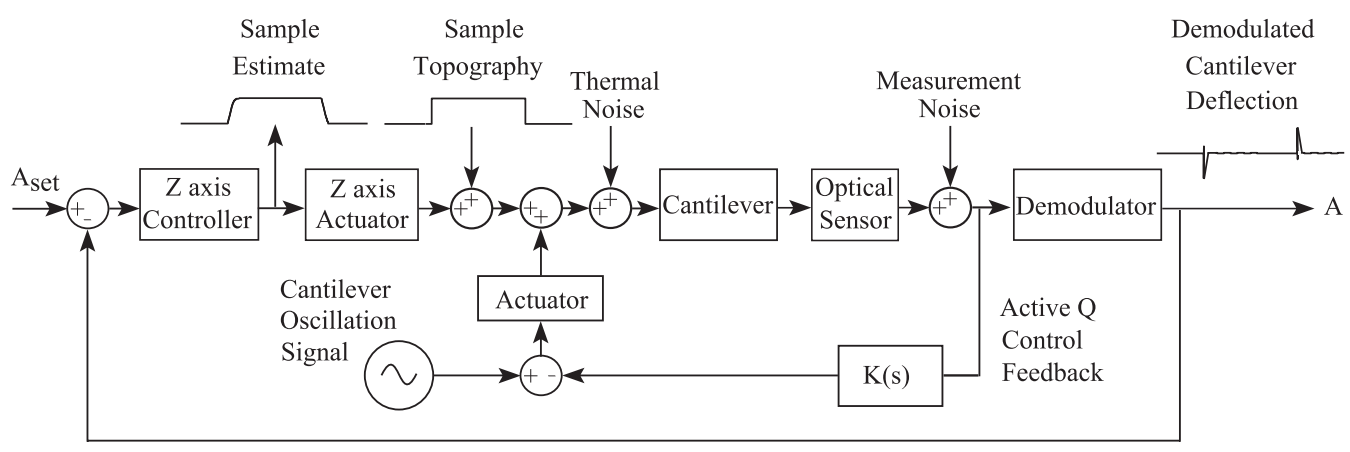

FIG. 2. Block diagram of the $Z$ axis feedback loop. $A$ is the oscillation amplitude of the cantilever and $A_{\text {set }}$ is the set point amplitude. An optional active $Q$ control feedback loop is also shown. 
requires negative feedback, whereas positive feedback is required to enhance the $Q$ factor.

The differential equation describing the motion of the cantilever, when the AFM is operating in tapping mode, is

$$
m \ddot{d}(t)+\frac{m \omega_{n}}{Q} \dot{d}(t)+k d(t)=A_{a} \cos \left(\omega_{o} t\right)+F_{T S}(t),
$$

where $m$ is the effective mass of the cantilever, $d$ is vertical tip displacement, and $k$ is the cantilever spring constant. Two external forces are acting on the cantilever. $A_{a} \cos \left(\omega_{o} t\right)$ is the force from the piezoelectric actuator with an oscillation amplitude of $A_{a}$ and an oscillation frequency of $\omega_{o} . F_{T S}(t)$ is the force due to tip-sample interaction.

From (2) it can be seen that the effective cantilever $Q$ factor $\left(Q^{*}\right)$ may be modified by adding an additional force proportional to the probe velocity via feedback. If the velocity signal is multiplied by a gain $G$ and subtracted from the probe actuation signal the cantilever equation of motion now becomes

$m \ddot{d}(t)+\frac{m \omega_{n}}{Q} \dot{d}(t)+k d(t)=A_{a} \cos \left(\omega_{o} t\right)+F_{T S}(t)-G \dot{d}(t)$,

or equivalently

$m \ddot{d}(t)+\left(\frac{m \omega_{n}}{Q}+G\right) \dot{d}(t)+k d(t)=A_{a} \cos \left(\omega_{o} t\right)+F_{T S}(t)$.

Which simplifies to

$$
m \ddot{d}(t)+\frac{m \omega_{n}}{Q^{*}} \dot{d}(t)+k d(t)=A_{a} \cos \left(\omega_{o} t\right)+F_{T S}(t),
$$

where

$$
Q^{*}=\frac{1}{\left(\frac{1}{Q}+\frac{G}{m \omega_{n}}\right)} .
$$

The effective $Q$ factor of the cantilever is decreased when the gain $G$ is positive and it is increased when the gain $G$ is negative.

The AFM is fitted with a displacement sensor. The addition of a velocity sensor would be difficult to implement in the AFM and would also add to the size and cost of the AFM. For these reasons cantilever velocity is most commonly estimated from the displacement signal.

The use of a differentiator to estimate velocity is not recommended as differentiators amplify high frequency noise in the feedback loop. The active $Q$ control feedback loop is influenced by two forms of noise as shown in Fig. 2. Thermal noise is due to surrounding molecules randomly hitting the cantilever and causing random movements. ${ }^{11}$ Thermal noise is modeled as an input to the cantilever in Fig. 2. The other form of noise is from the optical deflection measurement sensor which typically has a noise density of $100-1000 \mathrm{fm} / \sqrt{\mathrm{Hz}}$ (Ref. 30) in a commercial AFM.

An observer may be designed to estimate the probe velocity. ${ }^{31}$ The trade-off between observer gain, which determines the time that the observer accurately models the system state, and noise amplification may be an issue when implementing an observer for this application.
When active $Q$ control is applied to commercial AFMs it is most common to obtain an estimate of the cantilever velocity by applying a phase shift to the displacement signal using a time delay circuit. ${ }^{32}$ This phase shifted signal is then multiplied by a gain $G$ and the resulting signal is subtracted from the cantilever oscillation signal before being applied to the cantilever actuator. This arrangement is shown in the active $Q$ control feedback loop of Fig. 2 where

$$
K(s)=G e^{-T_{d} s} .
$$

In the above equation $T_{d}$ is the time delay required to estimate the velocity at the resonance frequency. As the displacement signal is sinusoidal a delay of $\frac{3 \pi}{2}$ radians is required to estimate the velocity signal. This is achieved by setting $T_{d}$ to $\frac{3}{4 f_{r 1}}$, where $f_{r 1}$ is the frequency of the first cantilever resonance mode. To increase the cantilever $Q$ factor $G$ must be negative. Hence, the required delay is $\frac{\pi}{2}$ radians. In this case $T_{d}$ should be set to $\frac{1}{4 f_{r 1}}$.

\section{DEGRADATION OF ACTIVE $Q$ CONTROL PERFORMANCE DUE TO UNMODELED DYNAMICS}

Flexible structures such as cantilevers have an infinite number of resonant modes. When designing an active $Q$ controller for an AFM micro-cantilever only the first resonance mode is modeled. When using a truncated model of a flexible structure to design a controller problems may arise if the unmodeled resonance modes are excited by the control action. This phenomenon is termed the spill-over effect. . $^{33,34}$

This spill-over effect may degrade the closed loop system response by shifting the poles of higher order modes closer to the $j \omega$ axis than the first mode, which is being controlled. To increase scan speed it is desirable to decrease the transient response time of the cantilever. This is achieved by decreasing the $Q$ factor of the cantilever's first resonance mode. In doing so it is possible that one of the higher order resonance modes may become excited by the control action and have its poles pushed closer to the $j \omega$ axis than the first mode. This will have the adverse effect of increasing the cantilever transient response time. If the control gain is high enough it may even push the poles of the higher order mode past the $j \omega$ axis making the system unstable.

When designing a controller to enhance the cantilever $Q$ factor the spill-over effect is not a problem as the poles of the first resonance mode are being pushed closer to the $j \omega$ axis by the controller and will remain to be the dominant poles of the system.

The time delay active $Q$ controller does not have guaranteed stability in the presence of unmodeled cantilever dynamics. Stark ${ }^{35}$ observed instability resulting from the spill-over effect when reducing the cantilever $Q$ factor with a time delay active $Q$ controller.

The phase delay of the time delay controller $K(s)$ increases as frequency increases. A frequency response of a typical time delay active $Q$ controller set to reduce the cantilever $Q$ factor is presented in Fig. 3. The delay of the controller at the cantilever resonance frequency is set to $T_{d}=\frac{3}{4 f_{r 1}}$ and the 


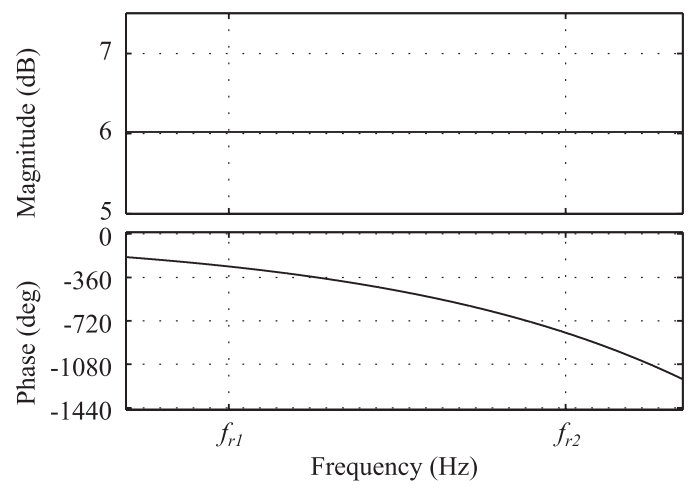

FIG. 3. Frequency response of the controller $K(s)=G e^{-T_{d} s}$ used to reduce the $Q$ factor of an AFM micro-cantilever with the active $Q$ control time delay method.

gain $G$ is set at an appropriate value to reduce the cantilever $Q$ factor by a desired amount. It can be seen from the frequency response of the cantilever given in Fig. 4 that the $Q$ factor of the first cantilever resonance mode has been decreased as desired. As a side effect of this reduction in $Q$ factor of the first resonance mode the $Q$ factor of the second resonance mode has increased. The reason for this is that the phase of the controller at the resonance frequency of the second mode $\left(f_{r 2}\right)$ is close to $-\frac{5 \pi}{2} \equiv-\frac{\pi}{2}$ which is the same as having a gain of $-G$. In this example $G$ is high enough to push poles of the second resonance mode into the right half of the complex plane.

If the phase delay of $K(s)$ at the resonance frequency of any of the higher order cantilever modes is close to $-2 \pi N$ $-\frac{\pi}{2}$ radians, for any integer $N$, the $Q$ factor of that mode will be enhanced. As $G$ is increased its poles will be pushed closer to the $j \omega$ axis. If $G$ is high enough the poles will cross the $j \omega$ axis making the system unstable.

\section{GUARANTEED STABILITY OF FEEDBACK SYSTEMS WITH UNMODELED DYNAMICS}

A useful technique for analyzing the closed loop stability of controllers for highly resonant systems with unmodeled

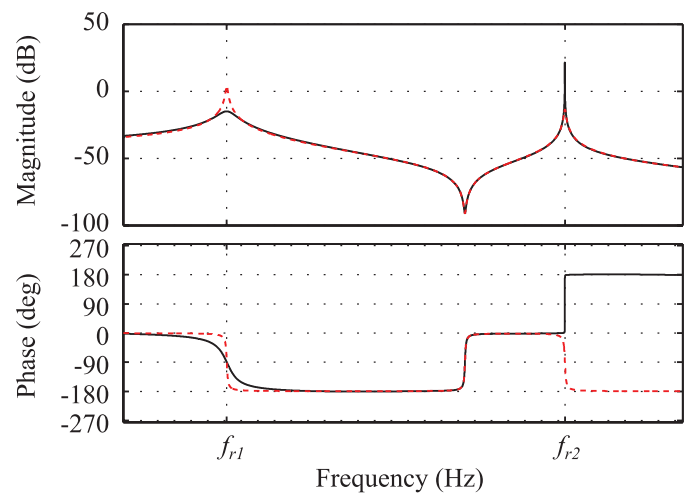

FIG. 4. A frequency response of a typical AFM micro-cantilever showing the first two resonant modes. (- -) The frequency response of the cantilever in open loop. (-) The frequency response of the cantilever with active $Q$ control using the time delay method. The controller effectively reduces the $Q$ factor of the first mode. As a side effect the $Q$ factor of the second mode is increased leading to instability of this mode. dynamics is the negative imaginary approach. ${ }^{36}$ When two negative imaginary systems are interconnected in a positive feedback loop, with at least one of the systems being strictly negative imaginary, closed loop stability is guaranteed if the dc loop gain is less than one. ${ }^{37}$

A negative imaginary transfer function is defined as a stable transfer function whose Nyquist plot for $\omega \geq 0$ lies on or below the real axis. A negative imaginary transfer function $M(s)$ satisfies the condition ${ }^{37}$

$$
j\left[M(j \omega)-M^{*}(j \omega)\right] \geq 0,
$$

for all $\omega \geq 0$.

A strictly negative imaginary transfer function defined as a stable transfer function whose Nyquist plot for $\omega>0$ lies below the real axis. A strictly negative imaginary transfer function $N(s)$ satisfies the condition ${ }^{37}$

$$
j\left[N(j \omega)-N^{*}(j \omega)\right]>0,
$$

for all $\omega>0$.

When the higher order modes of the cantilever are included in the model its transfer function, from a voltage applied to the piezoelectric actuator $V(s)$ to cantilever displacement $D(s)$, is

$$
G(s)=\frac{D(s)}{V(s)}=\sum_{i=0}^{\infty} \frac{\beta_{i} \omega_{i}^{2}}{s^{2}+2 \zeta_{i} \omega_{i} s+\omega_{i}^{2}},
$$

where $\omega_{i}$ is the natural frequency of the $i$ th mode, $\beta_{i}$ is the steady state gain of the $i$ th mode, and $\zeta_{i}$ is the damping factor of the $i$ th mode.

$$
j\left[G(j \omega)-G^{*}(j \omega)\right]=\sum_{i=0}^{\infty} \frac{4 \beta_{i} \zeta_{i} \omega_{i}^{3} \omega}{\left(\omega_{i}^{2}-\omega^{2}\right)^{2}+\left(2 \zeta_{i} \omega_{i} \omega\right)^{2}}
$$

is greater than 0 for all $\omega>0 . G(s)$ is therefore strictly negative imaginary as it is stable and satisfies condition (9).

To design a controller which guarantees closed loop stability when the model of the cantilever is truncated to a first mode approximation,

$$
G(s)=\frac{\beta \omega_{n}^{2}}{s^{2}+2 \zeta \omega_{n} s+\omega_{n}^{2}},
$$

the feedback structure of the active $Q$ control feedback loop is viewed in a positive feedback context by multiplying $K(s)$ by -1 as shown in Fig. 5 . The closed loop system will have guaranteed stability if $-K(s)$ is negative imaginary and the dc loop gain is less than one.

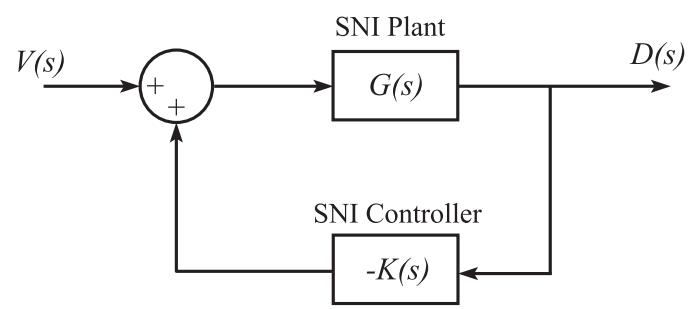

FIG. 5. Resonant control feedback loop arranged in a positive feedback context. The plant and controller both have strictly negative imaginary transfer functions. If the dc gain condition is satisfied then the closed loop system has guaranteed stability. 


\section{A RESONANT CONTROLLER FOR VELOCITY ESTIMATION IN ACTIVE $Q$ CONTROL}

In previous work the authors introduced passive piezoelectric shunt control ${ }^{19}$ to reduce the $Q$ factor of a piezoelectrically actuated bimorph micro-cantilever. When viewed in a positive feedback context the passive piezoelectric shunt controller is strictly negative imaginary and the dc closed loop gain of the system is less than one which ensures closed loop stability. Passive piezoelectric shunt control may not be applicable in all situations as the reduction of $Q$ factor is limited by the properties of the actuator and it may not be feasible to use a bimorph piezoelectric cantilever in some AFMs.

One controller which may be used in situations where passive piezoelectric shunt control is not feasible is the resonant controller. ${ }^{38}$ The similarities between passive piezoelectric shunt control and resonant control are discussed in detail in Ref. 39.

The method of resonant control has been developed to dampen vibrations in flexible structures. ${ }^{38,40,41}$ The resonant controller approximates a differentiator over a narrow range of frequencies. As a gain is applied only in the bandwidth of control the problem of high frequency noise being amplified by the differentiator is eliminated.

The transfer function of the resonant controller used in this work to modify the effective cantilever $Q$ factor is

$$
K(s)=\frac{\alpha s^{2}}{s^{2}+2 \zeta_{c} \omega_{*} s+\omega_{*}^{2}},
$$

where $\alpha$ and $\zeta_{c}$ are control design parameters which determine the gain at the frequency of interest $\left(\omega_{*}^{*}=\omega_{n}\right)$ and the bandwidth of control.

The resonant controller is implemented in the active $Q$ control feedback loop shown in Fig. 2. The input signal to the controller is the displacement measured by the photodiode sensor. The control signal is subtracted from the cantilever oscillation signal and then applied to the cantilever actuator.

To reduce the effective cantilever $Q$ factor with the resonant controller the value of $\alpha$ will always be positive. The frequency response of the controller when $\alpha$ is positive is shown in Fig. 6. As the phase of the controller is $\frac{\pi}{2} \mathrm{rad}$ at the cantilever's resonance frequency, it approximates a differentiator at this frequency. At higher frequencies the phase rolls off to

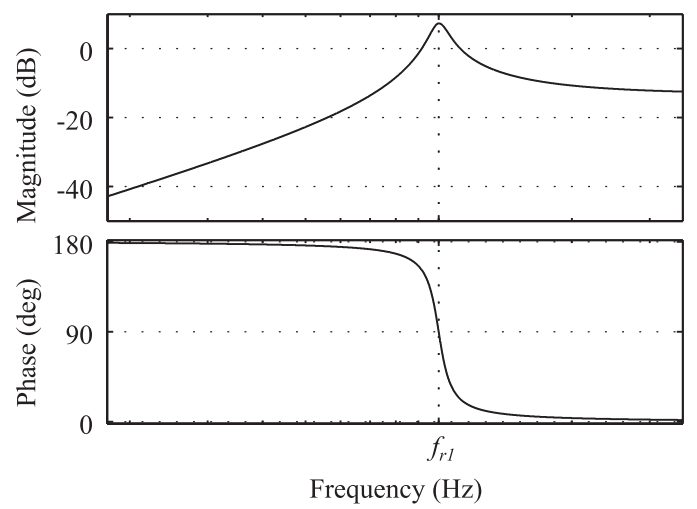

FIG. 6. Frequency response of the resonant controller $K(s)$ when $\alpha$ is positive $\left(\alpha=0.2190\right.$ and $\left.\zeta_{c}=0.0472\right)$.

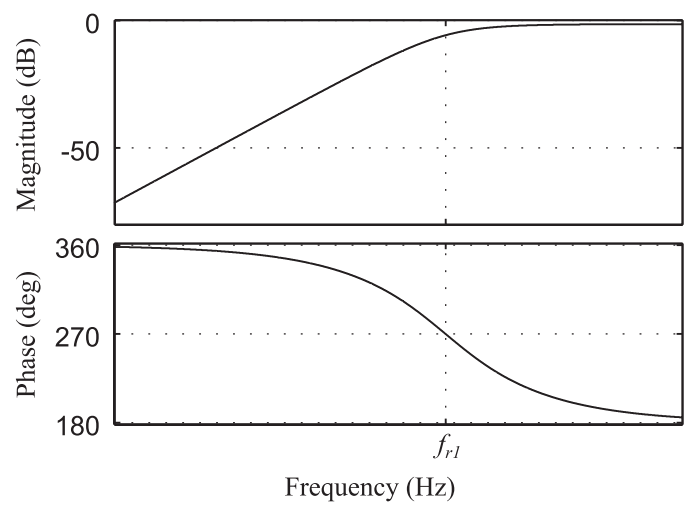

FIG. 7. Frequency response of the resonant controller $K(s)$ when $\alpha$ is negative $\left(\alpha=-0.8441\right.$ and $\left.\zeta_{c}=0.8263\right)$.

0 rad which ensures that the $Q$ factor of higher order modes of the cantilever are not enhanced. The poles of these higher order modes will not be pushed closer to the $j \omega$ axis.

If $\alpha$ is set to be negative the effective cantilever $Q$ factor is enhanced. The frequency response of the controller when $\alpha$ is negative is shown in Fig. 7. The phase at the cantilever's resonance frequency is now $-\frac{\pi}{2}$. This will have the effect of pushing the closed loop poles closer to the $j \omega$ axis, increasing the cantilever $Q$ factor.

The closed loop complementary sensitivity function, when the resonant controller is applied, is

$$
\begin{aligned}
T(s) & =\frac{D(s)}{V(s)}=\frac{G(s)}{1+G(s) K(s)} \\
& =\frac{\beta \omega_{n}^{2}\left(s^{2}+2 \zeta_{c} \omega_{n} s+\omega_{n}^{2}\right)}{\left(s^{2}+2 \zeta \omega_{n} s+\omega_{n}^{2}\right)\left(s^{2}+2 \zeta_{c} \omega_{n} s+\omega_{n}^{2}\right)+\beta \omega_{n}^{2} \alpha s^{2}} \\
& =\frac{\beta \omega_{n}^{2}\left(s^{2}+2 \zeta_{c} \omega_{n} s+\omega_{n}^{2}\right)}{s^{4}+\varphi s^{3}+\gamma s^{2}+\psi s+\omega_{n}^{4}},
\end{aligned}
$$

where $\varphi=2 \zeta \omega_{n}+2 \zeta_{c} \omega_{n}, \gamma=2 \omega_{n}^{2}+4 \zeta \zeta_{c} \omega_{n}^{2}+\beta \omega_{n}^{2} \alpha$, and $\psi=2 \zeta \omega_{n}^{3}+2 \zeta_{c} \omega_{n}^{3}$.

Through the technique of pole placement the values of $\alpha$ and $\zeta_{c}$ may be determined to place the poles of $T(s)$ in locations of the complex plane to obtain the desired cantilever $Q$ factor. Once the controller is initially tuned the operator may adjust the $Q$ factor by simply varying $\alpha$. Increasing $\alpha$ results in a decrease in $Q$ and reducing $\alpha$ results in an increase in $Q$.

\section{CLOSED LOOP STABILITY ANALYSIS OF THE RESONANT CONTROLLER}

To analyze the closed loop stability of $T(s)$, when $\alpha$ and $\zeta_{c}$ are set to decrease the cantilever $Q$ factor, the control loop is viewed in a positive feedback context by multiplying $K(s)$ by -1 as shown in Fig. 5. If $\bar{K}(s)=-K(s)$ then

$$
j\left[\bar{K}(j \omega)-\bar{K}^{*}(j \omega)\right]=\frac{4 \alpha \zeta_{c} \omega_{n} \omega^{3}}{\left(\omega_{n}^{2}-\omega^{2}\right)^{2}+\left(2 \zeta_{c} \omega_{n} \omega\right)^{2}}
$$

is greater than 0 for all $\omega>0 .-K(s)$ is therefore strictly negative imaginary as it is stable and satisfies condition (9).

As both of the transfer functions in the positive feedback loop of Fig. 5 are strictly negative imaginary the closed loop 
transfer function $T(s)$ will be guaranteed stable in the presence of the unmodeled dynamics of $G(s)$ if the dc loop gain is less than one, i.e.,

$$
G(0)(-K(0))<1,
$$

which holds true for $G(s)$ and $-K(s)$.

The negative imaginary approach cannot be used when increasing the cantilever $Q$ factor as $-K(s)$ is not strictly negative imaginary in this case. As mentioned previously the poles of the first cantilever resonance will remain the dominant poles when the $Q$ factor is increased. The poles of the first cantilever mode must be placed carefully to ensure sufficient stability margins.

\section{CONTROLLER IMPLEMENTATION}

The controller $K(s)$ may be implemented using standard analog components such as operational amplifiers and passive components, a digital signal processor (DSP) or a field programmable analog array (FPAA). ${ }^{42}$

FPAAs use a programmable array of switched capacitor filters to implement analog transfer functions. ${ }^{43}$ FPAAs have an advantage over DSP based devices in that there are no quantization or sampling rate issues due to the analog nature of the device. The only limitation is the capacitor switching frequency which is $16 \mathrm{MHz}$ for the AN221E04 FPAA (Ref. 44) used in this work which allows for a very high control bandwidth. The high resonance frequency of the cantilevers used in tapping mode limits the number of digital signal processor options for controller implementation due to the high sampling rate required.

The FPAA is programmed through a PC interface allowing changes to the implemented transfer function to be easily made. The ease in which control parameters may be changed gives the FPAA implementation an advantage over implementation with standard analog components.

The AN221E04 FPAA inputs and outputs are 0-4 V differential signals referenced to $2 \mathrm{~V}$. The use of differential signals reduces the amount of noise processed by the device. To convert and scale the ground referenced single ended signals used by the AFM an interface circuit was developed using LT 1468 operational amplifiers. ${ }^{45}$

\section{POLE PLACEMENT OPTIMIZATION TECHNIQUE FOR OBTAINING A DESIRED $Q$ FACTOR}

\section{A. Reducing the effective cantilever $Q$ factor}

For a second order system it is relatively easy to place the closed loop poles to achieve a desired $Q$ factor. The real part of the poles in the complex plane should be placed at

$$
\Re\left(p_{i}\right)=-\frac{\omega_{n}}{2 Q},
$$

where $\mathfrak{R}\left(p_{i}\right)$ is the real part of the $i$ th pole of the transfer function.

The closed loop transfer function $T(s)$ is a fourth order transfer function with a pair of complex conjugate zeros. Analytically determining the effective $Q$ factor of a higher order system such as $T(s)$ could be a tedious task. If all four poles of $T(s)$ are placed in the same location along the real axis, and the zeros are placed deeper into the left half plane than the poles, the frequency response of the closed loop system may be approximated by a second order system. In this case the approximate location of the poles of $T(s)$ along the real axis in relation to the effective $Q$ factor, $Q^{*}$ is

$$
\Re\left(p_{i}\right)=-\frac{\omega_{n}}{Q^{*}} .
$$

A pole placement optimization technique was used in this work to place the poles of $T(s)$ at desired locations along the real axis to obtain a desired effective $Q$ factor according to the relationship shown in (18). Only the desired real part of the poles of $T(s)$ is specified in the optimization rather than the exact location of the poles.

The resonant controller $K(s)$ has two parameters $\left(\alpha, \zeta_{c}\right)$ which may be adjusted to place the poles of $T(s)$ at desired locations to obtain a desired cantilever quality factor $Q_{d e s}$.

The cost function

$$
J\left(\alpha, \zeta_{c}\right)=\sum_{i=1}^{4}\left(\Re\left(p_{i}^{a c t}-p_{i}^{d e s}\right)\right)^{2},
$$

minimizes the absolute distance between the desired position of the poles of $T(s)$ along the real axis of the complex plane $\left(\Re\left(p_{i}^{d e s}\right)\right)$ and the actual position of the poles of $T(s)$ along the real axis $\left(\Re\left(p_{i}^{a c t}\right)\right)$. As $T(s)$ has four poles, the sum of absolute distance for each of the four poles is used.

The Matlab function fminsearch uses the Nelder-Mead algorithm ${ }^{46}$ to determine the unconstrained minimum of a given cost function and the values of the variables which produce this minimum. fminsearch $(J(\alpha$, $\left.\left.\zeta_{c}\right)\right)$ returns the values of $\alpha$ and $\zeta_{c}$ for which $J\left(\alpha, \zeta_{c}\right)$ is a minimum. As a result the poles of $T(s)$ will be placed as close as possible to the desired pole locations in order to achieve the desired cantilever $Q$ factor.

The AFM micro-cantilever used to demonstrate the effectiveness of the resonant controller in this work is the DMASP piezoelectric self-actuated micro-cantilever manufactured by Bruker. $^{47}$

A frequency response of $G(s)$ for the DMASP cantilever, shown in Fig. 8, was obtained using a Stanford Research Systems SRS780 dynamic signal analyzer. A periodic chirp signal of $1 \mathrm{~V}$ was applied to the cantilever and the voltage output

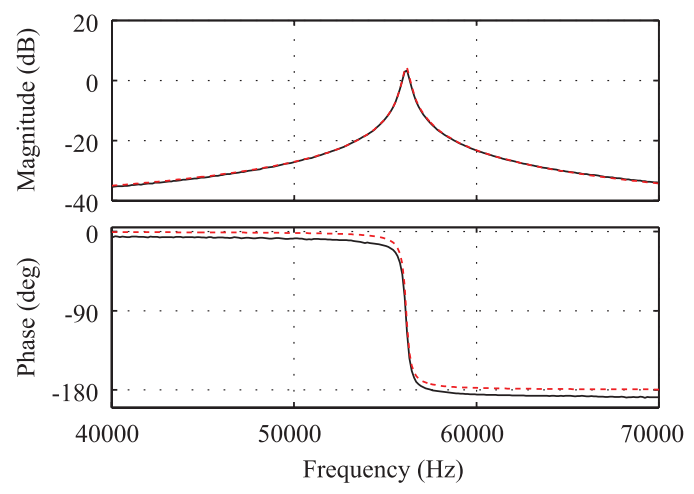

FIG. 8. Frequency response of DMASP cantilevers first resonance mode (一) and fitted model (- -). 


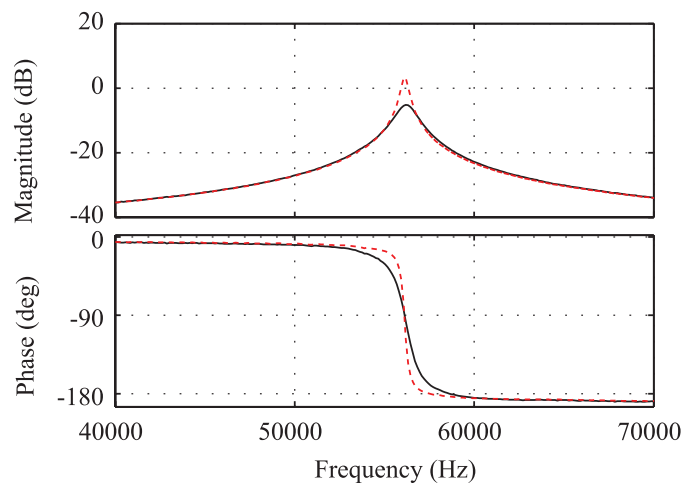

FIG. 9. Frequency response of the cantilever in open loop (- -), with a Q factor of 178.6, and closed loop (-) with an effective $Q$ factor of 37.5.

from the AFM photodiode sensor was recorded. The AFM used in this work is the NTEGRA AFM manufactured by NTMDT. $^{48}$ The transfer function

$$
G(s)=\frac{1.12 \times 10^{9}}{s^{2}+1976 s+1.245 \times 10^{11}}
$$

was obtained from the frequency response of Fig. 8 through system identification. From (20) the values for $\omega_{n}=2 \pi$ $\times 56150 \mathrm{rad}$ and $Q=178.6$ were obtained for the cantilever. $Q_{\text {des }}$ was chosen to be 40 , which gives a significant reduction in $Q$ while retaining sufficient force sensitivity.

The real part of the open loop poles is $\Re\left(p_{i}^{o l}\right)=\frac{\omega_{n}}{2 Q}$ $=-988$. The desired real part of the closed loop poles is $\Re\left(p_{i}^{d e s}\right)=\frac{\omega_{n}}{Q_{\text {des }}}=-8820$.

The pole placement optimization technique described above was used to place all four poles of $T(s)$ at $\Re\left(p_{i}^{d e s}\right)$. The optimal values for $\alpha$ and $\zeta_{c}$ were found to be $\alpha=0.2190$ and $\zeta_{c}=0.0472$.

The controller was implemented in the FPAA with the parameters determined above for a desired $Q$ factor of 40 . The measured closed loop frequency response of $T(s)$ along with the measured open loop frequency response are shown in Fig. 9 and a pole zero map showing the open and closed loop pole locations is shown in Fig. 10. The closed loop pole locations in the real axis are very close to the desired poles of $\Re\left(p_{i}^{\text {des }}\right)=-8820$.

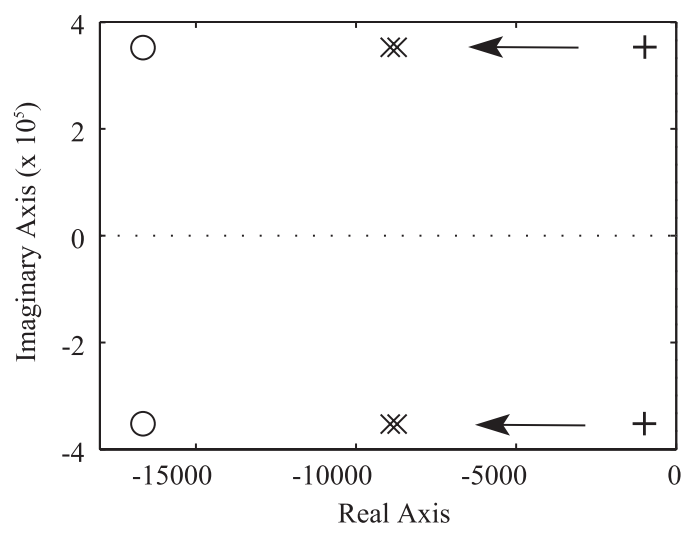

FIG. 10. The open loop pole (+) locations and closed loop pole (x) and zero (o) locations of the cantilever with resonant control.

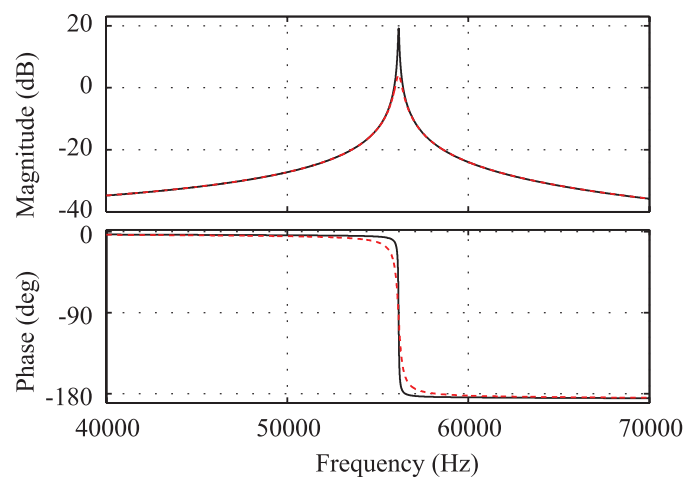

FIG. 11. Frequency response of the cantilever in open loop (- -), with a $Q$ factor of 178.6, and closed loop (-) with an effective $Q$ factor of 990.

The effective $Q$ factor of the closed loop system was determined from the frequency response of $T(s)$ in Fig. 9 by measuring the resonance frequency $f_{r}$ and the half power bandwidth $\Delta f_{-3 \mathrm{~dB}}$. The effective $Q$ factor is

$$
Q^{*} \cong \frac{f_{r}}{\Delta f_{-3 \mathrm{~dB}}}
$$

The effective $Q$ factor measured from the frequency response of $T(s)$ in Fig. 9 using this method was 37.5.

\section{B. Increasing the effective cantilever $Q$ factor}

When increasing the cantilever $Q$ factor it is not possible to place all four poles of $T(s)$ at the desired locations close to the $j \omega$ axis as the cost function of (19) does not converge under feedback control with a controller structure of (13). It is only possible to place two of the poles of $T(s)$ at the desired locations. As these two poles will be the dominant poles of the closed loop transfer function the closed loop transfer function may be approximated by a second order model and the location of the dominant poles along the real axis is determined by (17).

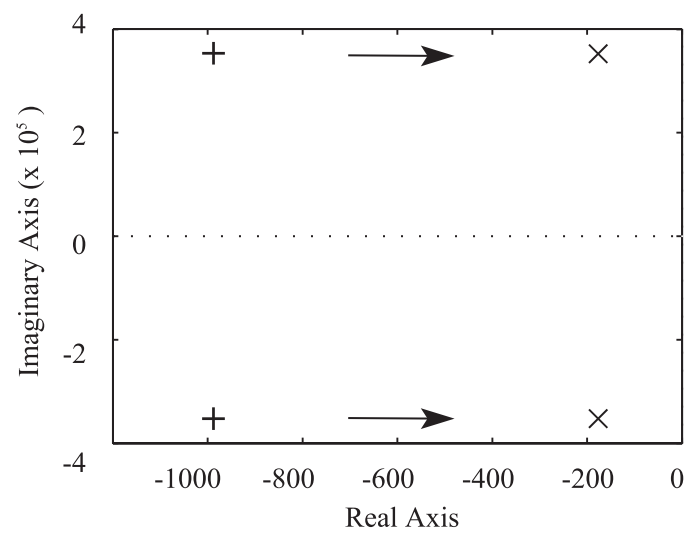

FIG. 12. The open loop pole (+) locations and closed loop pole (x) locations of the cantilever with resonant control $Q$ factor enhancement. Note that there is another pair of complex poles located at $-292000 \pm 198000 j$ in closed loop. 


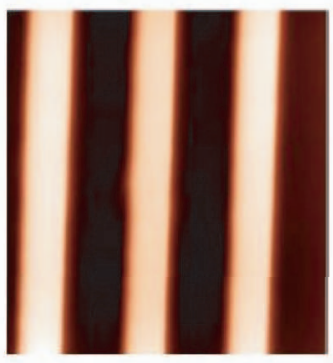

(a) 2D image without resonant control.

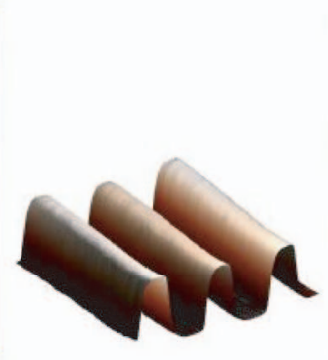

(b) 3D image without resonant control.

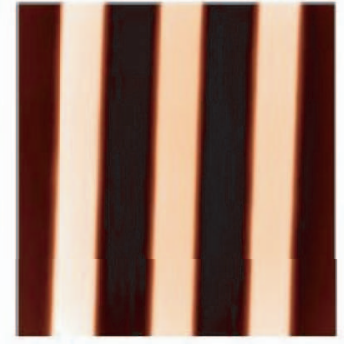

(c) $2 \mathrm{D}$ image with resonant control

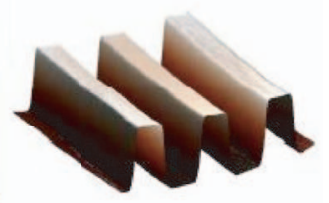

(d) 3D image with resonant control.

FIG. 13. Images of the NT-MDT TGZ1 calibration grating obtained at a scan speed of $20 \mu \mathrm{m} / \mathrm{s}$. In each case the maximum value of $K_{Z}$, which ensured loop stability, was used. The use of resonant control to damp the cantilever increased the cantilever response speed which allowed a higher feedback gain to be used and consequently reduced image distortion.

The cost function is modified to

$$
J\left(\alpha, \zeta_{c}\right)=\sum_{i=1}^{2}\left(\Re\left(p_{i}^{a c t}-p_{i}^{d e s}\right)\right)^{2},
$$

where the absolute distance between the desired position of two of the poles of $T(s)$ and the actual position of the poles of $T(s)$ along the real axis is minimized.

The pole placement technique was used to increase the cantilever $Q$ factor from $Q=178.6$ to $Q_{\text {des }}=1000$, where $\Re\left(p_{i}^{\text {des }}\right)$ is now 176.4. The values obtained for $\alpha$ and $\zeta_{c}$ using this technique place the remaining two poles of $T(s)$ deep into the left half of the complex plane. The optimal values for $\alpha$ and $\zeta_{c}$ were found to be $\alpha=-0.8441$ and $\zeta_{c}=0.8263$.

The open and closed loop frequency responses are shown in Fig. 11 and a pole zero map showing the open and closed loop pole locations is shown in Fig. 12. Note that only two of the closed loop poles are shown in the closed loop pole zero map. The remaining two closed loop poles are deep in the left half plane at $-292000 \pm 198000 j$. These poles may therefore be ignored. The poles shown on the pole zero map are at $-176 \pm 353000 j$. The effective cantilever $Q$ factor of $T(s)$ was measured at 990 from the frequency response of Fig. 11.

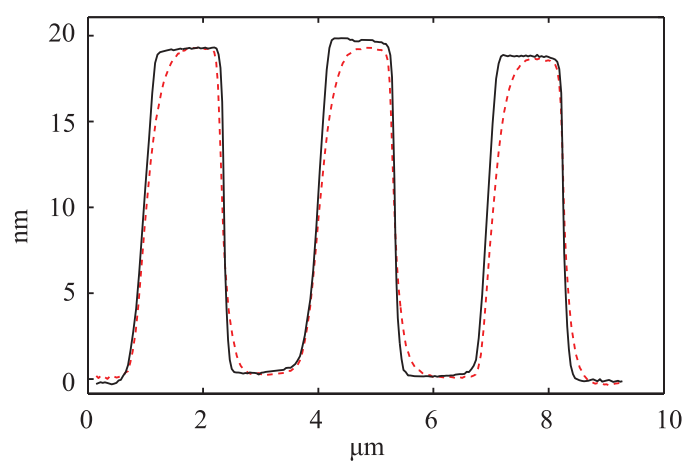

FIG. 14. Cross section of the NT-MDT TGZ1 sample topography obtained with (-) and without (- -) resonant control.

\section{Modification of the effective cantilever $Q$ factor after initial pole placement}

After the values of $\alpha$ and $\zeta_{c}$ have been set it is easy to modify the effective $Q$ factor. From (6) it can be seen that the effective $Q$ factor depends on the gain of the velocity feedback. This gain is the value of $K(s)$ at $\omega_{n}$ which is proportional to $\alpha$. Increasing $\alpha$ will reduce the effective $Q$ factor and decreasing $\alpha$ will enhance the effective cantilever $Q$ factor.

\section{AFM IMAGING WITH THE RESONANT CONTROL TECHNIQUE}

To demonstrate the effectiveness of the resonant controller and the benefits of reducing the cantilever $Q$ factor, images of a NT-MDT TGZ1 (Ref. 48) calibration grating were obtained with and without the resonant controller. The grating consists of a periodic step formed from silicon dioxide with a period of $3 \pm 0.05 \mu \mathrm{m}$ and step height of $18.5 \pm 1 \mathrm{~nm}$.

Scans were obtained on a $10 \mu \mathrm{m} \times 10 \mu \mathrm{m}$ section of the calibration grating at a scan speed of $20 \mu \mathrm{m} / \mathrm{s}$. Images were obtained with no $Q$ control $(Q=178.6)$ and with the resonant controller applied to reduce the cantilever $Q$ factor to $Q=37.5$. The $Z$ axis feedback controller gain $\left(K_{Z}\right)$ was increased until the loop became unstable. $K_{Z}$ was then reduced slightly to ensure loop stability. The maximum value of $K_{Z}$ obtainable when the resonant controller was applied was 7 times the gain obtainable without the controller. The images obtained with and without the resonant controller are shown in Fig. 13. A cross section of the 3D images is shown in Fig. 14. It can be seen from these images that the increase in feedback gain significantly reduced the distortion of the image obtained. This demonstrates that using resonant control to reduce the cantilever effective $Q$ factor can significantly improve the $Z$ axis feedback bandwidth which improves image quality at high scan speeds.

\section{CONCLUSION}

This work has highlighted a potential problem when reducing the effective $Q$ factor of an AFM micro-cantilever with the commonly used time delay velocity estimation for active $Q$ control. It is possible that higher order modes of the 
cantilever may be excited by the control action leading to degradation of performance and possible instability.

A resonant controller designed to estimate velocity in the active $Q$ control feedback loop has been presented in this work. Like the time delay controller, the resonant controller estimates the cantilever tip velocity by phase shifting the measured cantilever displacement signal by 90 degrees at the oscillation frequency. The main advantage of using the resonant controller, when reducing the cantilever $Q$ factor to improve the image quality at high scan speeds, is that it guarantees closed loop stability in the presence of unmodeled higher order cantilever dynamics. It has been shown that the controller may also be used to increase the cantilever $Q$ factor if increased force sensitivity and reduced tapping forces are desired.

By implementing the controller with a FPAA it has been demonstrated that the control system may be designed to be simple, compact and straightforward to tune. Initial tuning may be achieved through a pole placement technique, then the cantilever $Q$ factor may be adjusted by varying the controller gain.

The effectiveness of the controller has been demonstrated by reducing the effective cantilever $Q$ factor with the resonant controller and imaging a calibration grating with the AFM. A significant reduction in imaging artifacts when imaging at high scan speeds was observed when reducing the $Q$ factor as expected.

${ }^{1}$ D. Rugar and P. Hansma, Phys. Today 43, 23 (1990).

${ }^{2}$ T. Hassenkam, G. E. Fantner, J. A. Cutroni, J. C. Weaver, D. E. Morse, and P. K. Hansma, Bone 35, 4 (2004).

${ }^{3}$ F. J. Giessibl, Rev. Mod. Phys. 75, 949 (2003).

${ }^{4}$ Z. Shao and J. Yang, Q. Rev. Biophys. 28, 195 (1995).

${ }^{5}$ R. Garcia and R. Perez, Surf. Sci. Rep. 47, 197 (2002).

${ }^{6}$ B. Rogers, L. Manning, T. Sulchek, and J. Adams, Ultramicroscopy 100, 267 (2004).

${ }^{7}$ C. A. J. Putman, K. O. V. der Werf, B. G. D. Grooth, N. F. V. Hulst, and J. Greve, Appl. Phys. Lett. 64, 2454 (1994).

${ }^{8}$ N. C. Santos and M. A. Castanho, Biophys. Chem. 107, 133 (2004)

${ }^{9}$ A. Alessandrini and P. Facci, Meas. Sci. Technol. 16, R65 (2005).

${ }^{10}$ A. D. L. Humphris, J. Tamayo, and M. J. Miles, Langmuir 16, 7891 (2000).

${ }^{11}$ J. Tamayo, J. Appl. Phys. 97, 044903 (2005).

${ }^{12}$ G. Meyer and N. M. Amer, Appl. Phys. Lett. 53, 1045 (1988).

${ }^{13}$ J. Mertz, O. Marti, and J. Mlynek, Appl. Phys. Lett. 62, 2344 (1993).

${ }^{14}$ D. A. Walters, J. P. Cleveland, N. H. Thomson, P. K. Hansma, M. A. Wendman, G. Gurley, and V. Elings, Rev. Sci. Instrum. 67, 3583 (1996).

${ }^{15}$ T. Ando, Nanotechnology 23, 062001 (2012).

${ }^{16} \mathrm{H}$. Holscher and U. D. Schwarz, Int. J. Non-Linear Mech. 42, 608 (2007).
${ }^{17}$ T. Sulchek, R. Hsieh, J. D. Adams, G. G. Yaralioglu, S. C. Minne, C. F. Quate, J. P. Cleveland, A. Atalar, and D. M. Adderton, Appl. Phys. Lett. 76, 1473 (2000).

${ }^{18}$ T. Sulchek, G. G. Yaralioglu, C. F. Quate, and S. C. Minne, Rev. Sci. Instrum. 73, 2928 (2002).

${ }^{19}$ M. W. Fairbairn, S. O. R. Moheimani, and A. J. Fleming, IEEE/ASME J. Microelectromech. Syst. 20, 1372 (2011).

${ }^{20}$ F. D. Callaghan, X. Yu, and C. J. Mellor, Appl. Phys. Lett. 81, 916 (2002).

${ }^{21}$ F. Gittes and C. F. Schmidt, Eur. Biophys. J. Biophys. Lett. 27, 75 (1998).

${ }^{22}$ J. Tamayo, A. D. L. Humphris, and M. J. Miles, Appl. Phys. Lett. 77, 582 (2000).

${ }^{23}$ R. D. Jaggi, A. Franco-Obregon, P. Studerus, and K. Ensslin, Appl. Phys. Lett. 79, 135 (2001).

${ }^{24}$ T. R. Rodriguez and R. Garcia, Appl. Phys. Lett. 82, 4821 (2003).

${ }^{25}$ N. Kodera, M. Sakashita, and T. Ando, Rev. Sci. Instrum. 77, 083704 (2006).

${ }^{26}$ P. Agarwal, T. De, and M. V. Salapaka, Rev. Sci. Instrum. 80, 103701 (2009).

${ }^{27}$ J. Tamayo, A. D. L. Humphris, R. J. Owen, and M. J. Miles, Biophys. J. 81, 526 (2001).

${ }^{28}$ S. Gao, L. Chi, S. Lenhert, B. Anczykowski, C. M. Niemeyer, M. Adler, and H. Fuchs, ChemPhysChem 2, 384 (2001).

${ }^{29}$ L. Chen, X. Yu, and D. Wang, Ultramicroscopy 107, 275 (2007).

${ }^{30}$ S. Rode, R. Stark, J. Lubbe, L. Troger, J. Schutte, K. Umeda, K. Kobayashi, H. Yamada, and A. Kuhnle, Rev. Sci. Instrum. 82, 073703 (2011).

${ }^{31}$ D. R. Sahoo, T. De, and M. Salapaka, in Proceedings of the 44th IEEE Conference on Decision and Control, 2005 and 2005 European Control Conference, CDC-ECC'05, pp. 1185-1190 (2005).

${ }^{32}$ B. Bhushan, Springer Handbook of Nanotechnology, Gale Virtual Reference Library Vol. 2 (Springer, 2006).

${ }^{33}$ M. Balas, Automatic Control, IEEE Trans. 23, 673 (1978).

${ }^{34}$ J. Bontsema and R. Curtain, IEEE Trans. Autom. Control 33, 567 (1988).

${ }^{35}$ R. Stark, in Proceedings of the 5th IEEE Conference on Nanotechnology, Vol. 1, pp. 259-262 (2005).

${ }^{36}$ A. Lanzon and I. Petersen, IEEE Trans. Autom. Control 53, 1042 (2008).

${ }^{37}$ I. Petersen and A. Lanzon, IEEE Control Syst. 30, 54 (2010).

${ }^{38}$ H. R. Pota, S. O. R. Moheimani, and M. Smith, Smart Mater. Struct. 11, 1 (2002).

${ }^{39}$ S. O. R. Moheimani, A. J. Fleming, and S. Behrens, Smart Mater. Struct. 12, 49 (2003).

${ }^{40}$ S. O. R. Moheimani and B. J. G. Vautier, IEEE Trans. Control Syst. Technol. 13, 1021 (2005)

${ }^{41}$ I. A. Mahmood, S. O. R. Moheimani, and B. Bhikkaji, IEEE/ASME Trans. Mechatron. 13, 180 (2008).

${ }^{42} \mathrm{P}$. Gulak, in Proceedings of the IEEE Region 10 International Conference on Microelectronics and VLSI, TENCON'95, pp. 123-126 (1995).

${ }^{43}$ P. V. Swamy, M. N. S. Ananda Mohan, and V. Ramachandran, Switched Capacitor Filters: Theory, Analysis and Design (Prentice Hall, 1995).

${ }^{44}$ See http://www.anadigm.com/an220e04.asp for "Anadigm AN220E04 Data Sheet."

${ }^{45}$ See http://cds.linear.com/docs/Datasheet/1468fb.pdf for "Linear technology LT1468 datasheet."

${ }^{46}$ J. A. Nelder and R. Mead, Comput J. 7, 308 (1965).

${ }^{47}$ See http://www.brukerafmprobes.com/p-3252-dmasp.aspx for "Bruker DMASP cantilever specifications."

${ }^{48}$ See http://www.ntmdt.com for "ND-MDT nanotechnology instrumentation." 